\title{
Évaluation de l'impact de 6 matières grasses (palme et non-palme) sur les caractéristiques instrumentales et sensorielles d'une matrice de type cake
}

\author{
Sophie DELACHARLERIE ${ }^{1}$ \\ Céline PONCELET ${ }^{1}$ \\ Christine $\mathrm{CHENE}^{2}$ \\ Marianne SINDIC ${ }^{1}$ \\ ${ }^{1}$ Université de Liège - Gembloux Agro- \\ Bio Tech, \\ Laboratoire de Qualité et Sécurité des \\ Produits Agroalimentaires, \\ Passage des Déportés 2, \\ 5030 Gembloux, \\ Belgique \\ $<$ sdelacharlerie@ulg.ac.be $>$ \\ ${ }^{2}$ ADRIANOR, \\ ZI Est, \\ Rue Jacquart, \\ 62217 Tilloy-les-Mofflaines, \\ France
}

Les industries agro-alimentaires ont récemment été confrontées aux exigences de certains distributeurs leur demandant de supprimer l'huile de palme de leurs produits. Cette exigence se fonde principalement sur deux arguments : I'argument environnemental (déforestation, émission de gaz à effet de serre, atteinte à la biodiversité) et l'argument nutritionnel (matière grasse saturée, riche en acide palmitique) (Greenpeace, 2007 ; Fitzherbert et al., 2008 ; Koh et Wilcove, 2008 ; Walrand et al., 2010 ; de Moraes Mizurini et al., 2011).

Les difficultés technologiques constituent un frein majeur à la substitution de l'huile de palme. En effet, de nombreuses formulations de produits ont été développées pour une matière grasse qui apporte une fraction solide (Graille et Pina, 1999 ; Berger et Idris, 2005 ; Kazier,

\begin{abstract}
Effect of 6 oils and fats (palm and no-palm) on cake texture and sensory characteristics

Six types of fats (oils, shortenings and margarines) from different vegetable (hydrogenated or not) or animal origins have been studied in a cake model. Cakes have been characterized through Texture Profile Analysis and sensory evaluation. Texture analysis shows that studied fats can be classified in two main categories, giving different texture characteristics: liquid oils on one hand and fats containing a solid fraction on the other. Descriptive sensory evaluation leads to the same conclusions. On the contrary, hedonic sensory evaluation shows no difference between the different fats. The results in this paper indicate also that shortenings classical solid fat content can be reduced, without impact on the final product characteristics. This paper shows thus that the nutritional profile of bakery products can be improved, without sacrificing the sensory quality. Notably, the palm oil content (and thus the saturated fatty acids content) reduction of today shortenings could be an answer to nutritional and environmental concerns.
\end{abstract}

Key words: cake, texture profile analysis, oils, shortenings, solid fat content

2007). Cette fraction solide pourrait être apportée par d'autres matières grasses présentant une fraction solide (matières grasses animales; matières grasses végétales hydrogénées, fractionnées, interestérifiées; autres matières grasses végétales " exotiques" naturellement saturées...). Cependant, ces diverses solutions présentent toutes un ou plusieurs inconvénients parmi lesquels : coût élevé, impact sensoriel et sur la conservation, mauvaise image auprès du consommateur, déplacement du problème environnemental... Par ailleurs, d'un point de vue strictement nutritionnel, le gain santé du remplacement de l'huile de palme par d'autres matières grasses saturées reste discutable. De même, le recours à I'huile de palme durable ne résout pas le problème nutritionnel (Kummerow, 2009 ; Legrand, 2010).
La clé du problème nutritionnel réside plutôt dans l'amélioration du profil en acides gras des produits via l'optimisation de la composition des shortenings et dans l'allègement (réduction de la teneur en matière grasse) des produits finis.

Il pourrait donc être envisagé de remplacer l'huile de palme par des matières grasses insaturées, et donc liquides à température ambiante. Le recours à ces sources végétales pourrait contribuer à améliorer le profil nutritionnel des produits (diminution du taux d'acides gras saturés (AGS) au profit des acides gras (mono)insaturés $(A G(M) I)$ ), ainsi que le réclame par exemple la Charte PNNS (Plan national Nutrition Santé), de façon à permettre une alimentation respectant les recommandations nationales (Martin, 2001 ; Conseil Supérieur de la Santé, 2009). Par contre, les rende-

Pour citer cet article : Delacharlerie S, Poncelet C, Chene C, Sindic M. Évaluation de l'impact de 6 matières grasses (palme et non-palme) sur les caractéristiques instrumentales et sensorielles d'une matrice de type cake. OCL $2012 ; 19$ (2) : 101-110. doi : 10.1684/ocl.2012.0434 
ments des cultures concernées étant inférieurs à celui du palmier à huile, le gain environnemental n'est pas évident (Corley, 2009). De plus, le passage à d'autres types de corps gras qui n'apporteraient pas de fraction solide entraînerait une révision en profondeur des formulations et des process, et probablement une modification sensible de certains produits.

Pour tenter de résoudre ces problèmes, de nouvelles solutions ont été développées et commencent à être disponibles sur le marché. II s'agit notamment d'huiles issues de sélections génétiques comme c'est le cas pour le tournesol " High Stearic High Oleic » (HSHO) qui présente une forte teneur à la fois en acide stéarique (saturé) et en acide oléique (insaturé) et offre donc une fraction solide, qui peut au besoin être isolée par fractionnement (Fernandez-Moya et al., 2005). De nouveaux systèmes dits " structurés " de type organogels, à base d'huiles liquides, et dont la consistance provient plus d'un phénomène de gélification que de cristallisation font actuellement l'objet de nombreuses recherches (Pernetti et al., 2007 ; Rogers, 2009).

Devant cette liste d'arguments parfois contradictoires, et pas toujours objectifs, il a donc paru utile de réaliser une étude comparative des différentes solutions citées ci-dessus afin d'en évaluer les avantages et inconvénients technologiques et sensoriels. L'objectif premier de cet article est donc de comparer dans une même étude I'huile de palme et quelques-uns de ses substituts potentiels. Les travaux ont également permis d'obtenir des résultats sur des solutions récentes pour lesquelles un manque de données objectives (indépendantes du fournisseur) avait été constaté.

Pour ce faire, un modèle " cake " a été choisi. En effet, la présence d'une fraction solide est particulièrement critique pour ce genre d'application (Bennion et Bamford, 1997 ; Cauvain et Young, 2006). Par ailleurs, la mise en œuvre de ce modèle est assez facile. Le but ici n'est pas de proposer une formulation optimisée. C'est pourquoi le choix de la recette utilisée s'est porté sur une composition relativement simple (ingrédients "de base " uniquement, sans améliorants ni additifs).

Au vu des nombreux rôles joués par la matière grasse dans la matrice étudiée, l'observation de différences entre les matières grasses testées est attendue a priori. Le but de cette étude est donc de décrire et de quantifier ces différences de façon objective, mais aussi d'évaluer leur impact sur l'appréciation du consommateur. Si une fraction solide est effectivement indispensable pour retrouver la texture qui serait obtenue avec I'huile de palme, est-elle indispensable pour satisfaire le consommateur? Les teneurs en fraction solide (solid fat content - SFC) présentées par les shortenings actuels sont relativement élevées : est-il indispensable de conserver une valeur aussi élevée, ou peut-on diminuer le taux de saturation et donc le SFC tout en conservant de bonnes caractéristiques du produit fini ?

\section{Matériel et méthodes}

\section{Matières grasses étudiées}

Six matières grasses, disponibles sur le marché, ont été étudiées : une huile de colza (Vandemoortele, Izegem, Belgique) qui constitue la référence " huile liquide ", un shortening à base d'huiles de palme, colza et coco ("Finor Rose NH NC", CSM, Merksem, Belgique) qui constitue la référence "palme", une huile de tournesol HSHO ("Nutrisun », Advanta, Buenos Aires, Argentine), une huile de colza épaissie avec des mono- et di-glycérides ("Finor Liquid C ", CSM, Merksem, Belgique - vendue initialement comme "bread improver"), une margarine à base d'huiles de tournesol, colza et coco hydrogénées et en l'état ("Tulip Rouge non palm», Unipro, Merksem, Belgique) et une margarine à base de matière grasse laitière et $d^{\prime}$ 'huile de colza ("Selection Rouge non palm ", Unipro, Merksem, Belgique). Ces matières grasses ont été stockées à $12{ }^{\circ} \mathrm{C}$ pendant toute la durée de l'étude.

Dans la suite de cet article, les échantillons sont nommés respectivement HC (huile de colza), SP (shortening palme), HSHO (tournesol HSHO), CMDG (colza + mono- et di- glycérides), MGVH (margarine graisses végétales hydrogénées) et MGVL (margarine graisses végétale et laitière).

\section{Fabrication et conservation des cakes}

La recette utilisée pour la fabrication des cakes est la suivante : farine blanche de blé type $000(23,4 \%)$, matière grasse
(14\%), sucre cristallisé (20\%), œufs entiers $(18,7 \%)$, eau (14\%), sirop de glucose $(6,7 \%)$, poudre de lait écrémé $(1,6 \%)$, poudre à lever $(1 \%)$, sel (0,6 \%) (Bennion et Bamford, 1997 ; Cauvain et Young, 2006).

Le mélange des ingrédients est réalisé au moyen d'un batteur Kenwood (Kenwood Chef - Modèle A910D) équipé d'un fouet. Le sirop de glucose est d'abord dilué dans l'eau préalablement portée à ébullition. Après refroidissement du sirop de glucose dilué, les œufs sont ajoutés et battus (1 minute, $240 \mathrm{rpm}$ ). Les ingrédients secs, préalablement mélangés, sont incorporés graduellement. La matière grasse est ajoutée en dernier lieu après homogénéisation manuelle. Le tout est battu 4 minutes (300 rpm).

Les corps gras liquides ou semi-solides (HC, HSHO et CMDG) sont incorporés à $12{ }^{\circ} \mathrm{C}$ (température de stockage). Les corps gras solides (SP, MGVH et MGVL) sont placés dans un bain-marie (GFL ; Modèle 1.4) à $40{ }^{\circ} \mathrm{C}$ préalablement à leur incorporation de façon à obtenir une texture souple (pommade). La durée du séjour dans le bain-marie est différente pour chaque matière grasse (MGVL: 30 minutes; MGVH : 1 h ; SP : 1 h 20). La température finale de ces matières grasses avant leur incorporation est vérifiée et est respectivement de 32, 35 et $37^{\circ} \mathrm{C}$. La température de la pâte avant l'incorporation de la matière grasse est également mesurée et est en moyenne de $24{ }^{\circ} \mathrm{C}$ (Bennion et Bamford, 1997).

La pâte est ensuite versée $(650 \mathrm{~g} /$ moule) dans des moules métalliques $(7 \times 8 \times 27 \mathrm{~cm})$ huilés avec environ $2,5 \mathrm{~g} \mathrm{~d}^{\prime}$ huile d'arachide et dont le fond a été recouvert de papier de cuisson. La cuisson est réalisée à $160^{\circ} \mathrm{C}$ durant $1 \mathrm{~h} 15$ (four de boulangerie modulaire Salva). Les cakes sont recouverts d'une feuille d'aluminium après 45 minutes de cuisson afin d'éviter un brunissement trop important de la croûte. Les cakes sont démoulés après refroidissement (soit environ $3 \mathrm{~h} 30$ après la fin de la cuisson).

Les cakes sont conditionnés le lendemain de leur fabrication sous atmosphère modifiée (ALIGAL 15, Air Liquide, Liège, Belgique) et avec un absorbeur d'oxygène (Absorbeur ATCO, Standa, Caen, France) dans des sachets PA/ EVOH/PA/PE (Colamin Multi Layer, EuralPack, Scoten; Belgique) choisis pour 
leurs faibles perméabilités à l'oxygène $\left(<2,7 \mathrm{~cm}^{3} / \mathrm{m}^{2}\right.$.j.bar), à la vapeur $\mathrm{d}^{\prime}$ eau $\left(<1,8 \mathrm{~g} / \mathrm{m}^{2}\right.$.j) et au $\mathrm{CO}_{2}\left(<6,5 \mathrm{~cm}^{3} / \mathrm{m}^{2}\right.$. j.bar). Le conditionnement est réalisé à I'aide d'une thermosoudeuse (Multivac ; A300/16). Les cakes sont stockés à $20^{\circ} \mathrm{C}$ dans une enceinte thermostatée (Memmert ; ICP 800).

\section{Dispositif expérimental}

Trois séries de fabrications sont réalisées, espacées dans le temps d'environ 2 semaines. Les cakes sont caractérisés dans leur état frais (le lendemain de leur fabrication) et après 3 mois de conservation. Les trois séries sont caractérisées de manière instrumentale, deux d'entre elles sont en plus soumises à l'analyse sensorielle descriptive et la troisième est utilisée pour l'analyse sensorielle hédonique.

\section{Caractérisation des matieres grasses}

Le profil de fusion de chaque matière grasse est réalisé par résonance magnétique nucléaire pulsée ( $R M N-p)$ à l'aide d'un spectromètre Minispec-mq 20 (Bruker) selon la méthode IUPAC 2.150. Les différentes matières grasses sont introduites dans des tubes pour RMN (Bruker, UK). Pour cela, les matières grasses solides sont prélevées par carottage. Les tubes sont ensuite placés à $70^{\circ} \mathrm{C}$ jusqu'à fusion complète de toutes les matières grasses, homogénéisés (agitation vortex) puis placés à $0{ }^{\circ} \mathrm{C}$ pendant 1 heure afin de permettre leur recristallisation. Trois tubes sont préparés pour chaque matière grasse. Avant mesure, les tubes sont placés 30 minutes dans un bain thermostatique (PolyScience; Modèle 910) afin d'atteindre la température désirée. Le SFC (Solid Fat Content) est mesuré à $5,10,15,20,25,30,35,40$, 45 et $50^{\circ} \mathrm{C}$.

\section{Caractérisation instrumentale des cakes}

Les cakes sont tranchés à l'aide d'une trancheuse électrique (modèle ménager de marque Krups) en tranches de $1,25 \mathrm{~cm}$ d'épaisseur.

Le développement des cakes est évalué via la hauteur de la tranche centrale (Cauvain et Young, 2006 ; Dogan et al., 2007). La mesure est effectuée à l'aide d'une règle graduée et relevée à l'extrême gauche, au centre et à l'extrême droite de la tranche.

La couleur de la mie est évaluée via les paramètres $L^{*} a * b *$ mesurés à l'aide $d^{\prime}$ un colorimètre Miniscan Y-2500 (Hunterlab Inc.). La mesure est effectuée sur trois tranches prélevées en différents endroits du cake (une mesure par tranche).

Trois autres tranches sont prélevées dans les mêmes zones que pour la mesure de la couleur puis broyées en vue de la mesure de l'activité d'eau et de la teneur en matière sèche. Le broyage est réalisé pendant 10 secondes à $10000 \mathrm{rpm}$ à l'aide d'un broyeur à couteaux (Retsch ; Grindomix GM200).

La teneur en matière sèche est déterminée par dessication à l'étuve (24 h à $103{ }^{\circ} \mathrm{C}$; Étuve Memmert ULM 500). Trois répétitions sont effectuées pour chaque échantillon.

L'activité d'eau $\left(a_{w}\right)$ est mesurée à l'aide d'un appareil de mesure Aqualab (Decagon; Modèle CX3) placé dans une chambre thermostatique à $20{ }^{\circ} \mathrm{C}$. Trois répétitions sont effectuées pour chaque échantillon.

La texture est mesurée grâce au texturomètre SMS TA-XT2 équipé d'une sonde cylindrique de $36 \mathrm{~mm}$ (Bourne, 2002), en mode TPA (Texture Profile Analysis) (tableau 1). Cing répétitions sont effectuées pour chaque cake analysé et sont réalisées sur 2 tranches superposées.

\section{Caractérisation sensorielle des cakes}

Afin d'établir les profils sensoriels des différents échantillons, un jury de 14 juges (âgés de 25 à 65 ans; dont 4 hommes et 10 femmes) a été recruté et entraîné. Les descripteurs sensoriels

Tableau 1. Paramètres utilisés pour l'analyse de texture

\begin{tabular}{|ll|}
\hline Paramètres & Valeurs \\
\hline Vitesse pré-test & $1 \mathrm{~mm} / \mathrm{s}$ \\
\hline Vitesse test & $1 \mathrm{~mm} / \mathrm{s}$ \\
\hline Vitesse post-test & $10 \mathrm{~mm} / \mathrm{s}$ \\
\hline Distance & $\begin{array}{l}6,2 \mathrm{~mm}(25 \% \\
\text { de compression })\end{array}$ \\
\hline Déclenchement & Auto $(0,05 \mathrm{~N})$ \\
\hline Temps & $10 \mathrm{~s}$ \\
\hline
\end{tabular}

ont été générés par le jury, puis sélectionnés, selon la norme AFNOR V09-021 (ISO 11035). Les descripteurs retenus sont I'aération de la mie, la couleur de la mie, le cassant, l'élasticité, la sensation d'humidité, la sensation de gras, le collant, la friabilité, la fermeté et la « mâchabilité » (Chueamchaitrakun et al., 2011). Les différents descripteurs sont notés sur des échelles discontinues graduées de 1 à 7. L'aération de la mie et la couleur sont évaluées visuellement, le cassant et l'élasticité sont mesurés à la main (toucher), les autres paramètres sont évalués en bouche (la fermeté à la première pression, les autres lors de la mastication). Lors de chaque séance, chaque juge reçoit 7 échantillons : 6 correspondent aux 6 matières grasses testées et 1 est issu du commerce et sert de contrôle. 10 des 14 juges sont présents à chacune des séances.

Parallèlement au jury entraîné, un jury initié (familiarisé à l'analyse sensorielle) de 34 personnes ( 15 hommes et 19 femmes âgés de 20 à 60 ans) a également été recruté. Ce jury a évalué les échantillons sous un angle hédonique. L'aspect visuel (couleur et aération de la mie), la texture, le goût, et l'appréciation globale du produit sont évalués de "très désagréable " à " très agréable " sur des échelles discontinues graduées de 1 à 7 . Lors de chaque séance, chaque juge a reçu 6 demitranches correspondant aux 6 matières grasses.

\section{Analyse statistique}

Les résultats sont analysés à l'aide du logiciel Minitab 15 EN (Minitab Inc., State College, Pennsylvanie, USA). Tous les paramètres étudiés ont été soumis à l'analyse de la variance (modèle général linéarisé ; $\alpha=0,05)$.

L'ANOVA II a été utilisée pour tous les paramètres ayant fait l'objet de plusieurs séries et ne pouvant a priori pas évoluer au cours du temps (couleur et hauteur par exemple). Pour ces paramètres, les données obtenues aux différents stades de conservation ont été traitées comme des répétitions d'un même point de mesure.

L'ANOVA III a été utilisée pour tous les paramètres ayant fait l'objet de plusieurs séries et ne pouvant pas être a priori déterminés comme stables au cours du temps (texture et activité d'eau par exemple). 
Les résultats de l'analyse sensorielle par le jury naif n'ayant pas fait l'objet de plusieurs séries, I'ANOVA I ou II a été utilisée selon qu'il s'agissait de paramètres pouvant ou non évoluer au cours du temps.

Le cas échéant, une structuration des moyennes a été réalisée selon la méthode de Newman et Keuls.

\section{Résultats et discussion}

D'après leur observation visuelle (figure 1), les cakes obtenus avec les huiles $\mathrm{HC}$ et $\mathrm{HSHO}$ se ressemblent, présentant une coloration similaire, un aspect bombé, et un développement légèrement plus important que les autres. Les tranches de ces cakes présentent également des colorations beige à brun très clair assez proches, une aération importante et un toucher élastique et spongieux. Le CMDG donne un cake relativement plat, peu aéré, jaune-orange, très humide, très fragile et s'émiettant fortement lors du tranchage. Les matières grasses solides (SP, MGVH et MGVL) donnent des cakes au développement intermédiaire et au profil plutôt plat, une coloration de mie blanche à jaune très pâle, et des tranches moins aérées, plus friables et

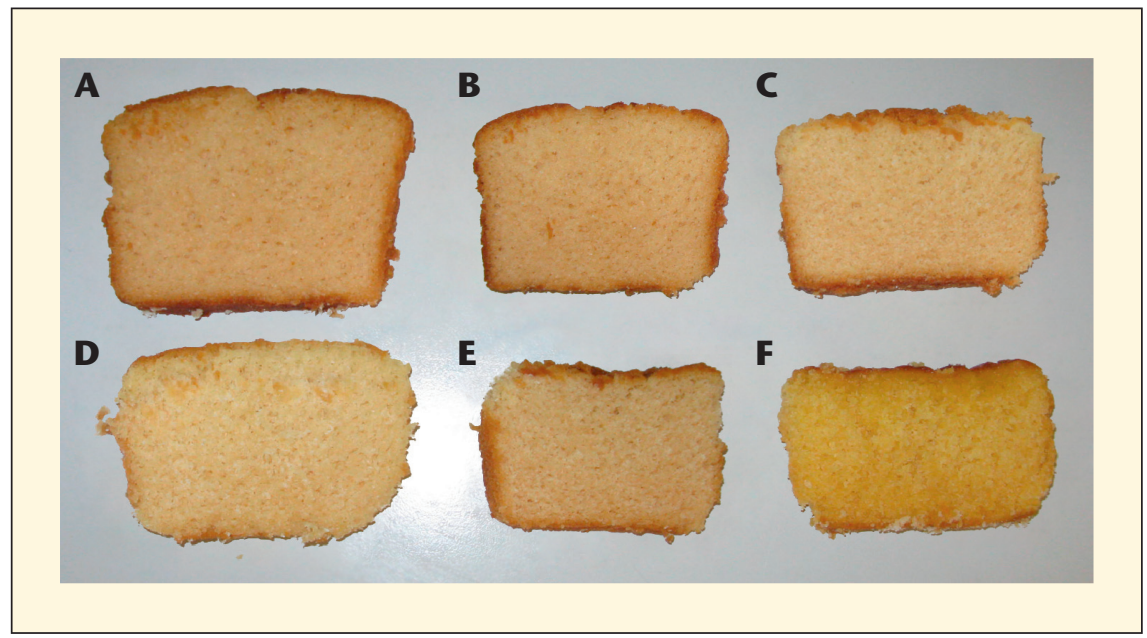

Figure 1. Tranches centrales des cakes obtenus avec A) HC, B) HSHO, C) MGVL, D) MGVH, E) $S P, F) C M D G$.

moins élastiques que les huiles qui développent une texture plutôt de type "sponge cake".

L'analyse statistique montre que les résultats instrumentaux ne sont pas significativement impactés par la série de fabrication. Aussi, pour un même stade de vieillissement, les résultats des différentes séries ont été traités globalement. Le nombre de répétitions repris dans les tableaux indique donc le nombre de cakes différents analysés (un par série de fabrication et stade de vieillissement).

Les résultats obtenus pour la hauteur de la tranche centrale et la couleur de la mie (tableau 2) ont été considérés a priori comme ne pouvant pas évoluer au cours du temps et sont donc calculés sur l'ensemble des résultats obtenus pour

Tableau 2. Hauteur et couleur des cakes obtenus (3 séries ; 2 durées de conservation) ${ }^{7}$

\begin{tabular}{|c|c|c|c|c|c|c|}
\hline \multirow{2}{*}{$n=6$} & \multicolumn{3}{|c|}{ Hauteur $(\mathrm{cm})$} & \multicolumn{3}{|c|}{ Couleur } \\
\hline & GAUCHE & CENTRE & DROITE & $\mathbf{L}^{*}$ & $A^{*}$ & B* $^{*}$ \\
\hline $\mathrm{HC}$ & $6,0^{a}$ & $6,3^{a}$ & $6,0^{a}$ & $67,6^{b}$ & $8,8^{c}$ & $35,9^{c}$ \\
\hline $\mathrm{HSHO}$ & $5,4^{b}$ & $5,9^{b}$ & $5,5^{b}$ & $64,7^{c}$ & $9,8^{b}$ & $37,3^{b}$ \\
\hline CMDG & $4,8^{d}$ & $5,3^{c}$ & $4,8^{d}$ & $65,4^{c}$ & $11,9^{a}$ & $46,6^{a}$ \\
\hline MGVL & $5,0^{\text {cd }}$ & $5,4^{c}$ & $5,1^{\mathrm{c}}$ & $72,4^{a}$ & $7,7^{\text {cd }}$ & $36,0^{b c}$ \\
\hline SP & $5,3^{b}$ & $5,3^{c}$ & $5,3^{b c}$ & $71,8^{\mathrm{a}}$ & $7,3^{d}$ & $33,4^{d}$ \\
\hline MGVH & $5,1^{b c}$ & $5,2^{c}$ & $5,1^{c}$ & $70,9^{a}$ & $7,8^{\mathrm{cd}}$ & $35,9^{c}$ \\
\hline
\end{tabular}

${ }^{1}$ Les différentes lettres en exposant indiquent, par colonne, les moyennes significativement différentes $(p=0,05)$

Tableau 3. Résultats de l'analyse de texture (TPA - 3 séries) ${ }^{1}$

\begin{tabular}{|c|c|c|c|c|c|c|c|c|}
\hline \multirow{2}{*}{$\mathbf{n}=\mathbf{3}$} & \multicolumn{2}{|c|}{ Dureté (N) } & \multicolumn{2}{|c|}{ Elasticité } & \multicolumn{2}{|c|}{ Gumminess (N) } & \multicolumn{2}{|c|}{ Cohésion } \\
\hline & Mois 0 & Mois 3 & Mois 0 & Mois 3 & Mois 0 & Mois 3 & Mois 0 & Mois 3 \\
\hline $\mathrm{HC}$ & $5,7^{\mathrm{a}}$ & $14,2^{\mathrm{ab}}$ & $0,87^{a}$ & $0,79^{a}$ & $3,1^{\mathrm{a}}$ & $7,1^{\mathrm{a}}$ & $0,54^{\mathrm{a}}$ & $0,50^{\mathrm{bc}}$ \\
\hline $\mathrm{HSHO}$ & $5,6^{\mathrm{a}}$ & $15,0^{a}$ & $0,83^{a}$ & $0,74^{a}$ & $3,0^{a}$ & $7,1^{\mathrm{a}}$ & $0,55^{a}$ & $0,51^{\mathrm{b}}$ \\
\hline CMDG & $2,7^{c}$ & $3,0^{c}$ & $0,71^{\mathrm{c}}$ & $0,60^{b}$ & $1,5^{c}$ & $1,7^{c}$ & $0,55^{\mathrm{a}}$ & $0,54^{a}$ \\
\hline MGVL & $5,3^{a b}$ & $14,3^{\mathrm{ab}}$ & $0,74^{\mathrm{bc}}$ & $0,60^{b}$ & $2,6^{\mathrm{b}}$ & $5,8^{b}$ & $0,51^{b}$ & $0,47^{c}$ \\
\hline SP & $5,1^{b}$ & $13,4^{b}$ & $0,76^{b}$ & $0,61^{b}$ & $2,6^{b}$ & $5,7^{b}$ & $0,51^{b}$ & $0,43^{d}$ \\
\hline MGVH & $5,1^{\mathrm{b}}$ & $13,9^{b}$ & $0,73^{b c}$ & $0,64^{b}$ & $2,7^{b}$ & $5,7^{b}$ & $0,53^{\mathrm{ab}}$ & $0,48^{\mathrm{c}}$ \\
\hline
\end{tabular}

${ }^{1}$ Les différentes lettres en exposant indiquent, par colonne, les moyennes significativement différentes $(p=0,05)$ 


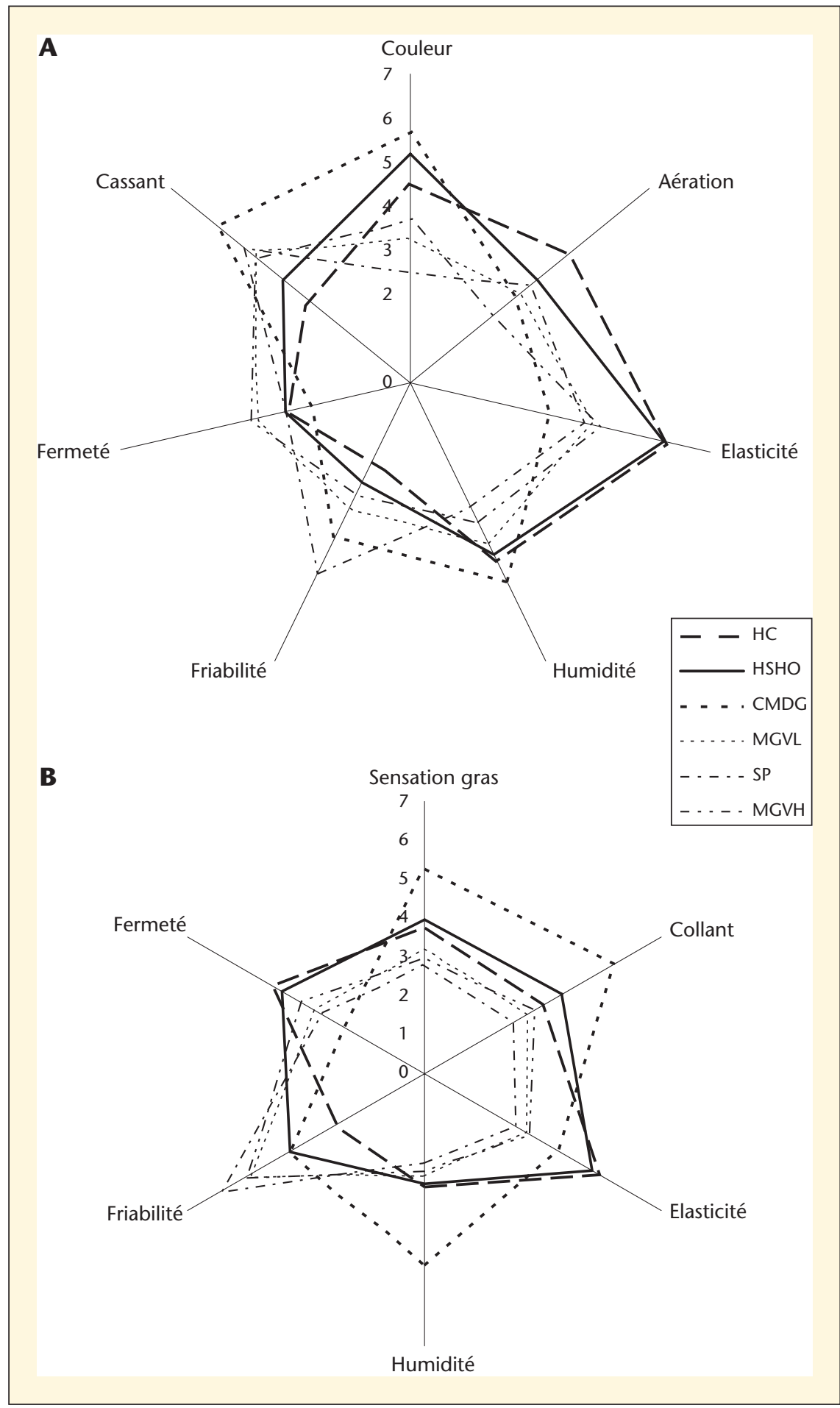

Figure 2. Profils sensoriels établis par le jury d'experts (2 séries; paramètres significatifs uniquement) pour les cakes $A$ ) frais, $B$ ) après 3 mois.

chaque matière grasse. La structuration des moyennes a montré que I'huile de colza $(\mathrm{HC})$ permettait d'obtenir le plus fort développement, suivi par le HSHO. Les trois matières grasses solides engen- grasses plutôt liquides entraînent un profil plutôt bombé tandis que les matières grasses solides donnent un profil plutôt plat. Le paramètre $L^{*}$ permet d'identifier deux groupes principaux avec d'un côté les trois matières grasses plutôt liquides qui donnent une mie légèrement plus sombre que les trois matières grasses solides. Les paramètres $a^{*}$ et $b^{*}$ permettent de rassembler les trois matières grasses solides qui présentent la même teinte, tandis que le $\mathrm{HC}$ et le $\mathrm{HSHO}$ ont une teinte légèrement différente. Le CMDG se classe à part : la mie obtenue avec cette matière grasse est en effet très jaune et cela s'explique par sa forte teneur en $\beta$-carotène.

L'activité d'eau est de 0,89 $\pm 0,01$ pour tous les cakes frais, n'est pas influencée par le type de matière grasse incorporée et n'évolue pas significativement au cours du temps dans les conditions de stockage appliquées pour cette étude. En revanche, comme on pouvait s'y attendre, les deux margarines (MGVH et MGVL) donnent une teneur en matière sèche un peu plus basse $(71,5 \pm 1,0 \%)$ que les quatre autres matières grasses qui ne contiennent pas d'eau $(73,7$ $\pm 1,1 \%)$. Au cours du stockage, la teneur en matière sèche augmente de 2-3\% pour toutes les matières grasses, excepté pour le CMDG. Ceci s'explique probablement par la forte teneur en émulsifiants de ce produit.

Le tableau 3 présente les résultats de l'analyse de texture. Aucune fracturabilité n'a été mesurée et l'adhérence n'a pas été systématiquement observée. Par ailleurs, les résultats obtenus pour le chewiness montrent les mêmes tendances que le gumminess. Ces trois paramètres ne sont donc pas présentés ici. L'analyse de texture permet de classer les échantillons en trois groupes : $\mathrm{HC}$ et HSHO montrent le même comportement, les trois matières grasses solides donnent des résultats identiques et le CMDG se classe à part. Ces conclusions sont avérées aussi bien sur les cakes frais qu'en fin de vieillissement et corroborent les observations réalisées visuellement. II est intéressant de souligner que, par rapport aux matières grasses solides, HC et $\mathrm{HSHO}$ donnent une texture plus élastique et un gumminess et une cohésion plus élevés, traduisant leur toucher spongieux. Le CMDG, quant à lui, confère une dureté et un gumminess nettement inférieurs aux autres matières grasses, et ces paramètres n'évoluent pas 
Tableau 4. Évolution des paramètres sensoriels au cours du stockage (2 séries; paramètres significatifs uniquement) ${ }^{7}$

\begin{tabular}{|c|c|c|c|c|c|c|c|c|}
\hline \multirow{2}{*}{$\mathbf{n}=\mathbf{2}$} & \multicolumn{2}{|l|}{ Élasticité } & \multicolumn{2}{|c|}{ Humidité } & \multicolumn{2}{|c|}{ Friabilité } & \multicolumn{2}{|c|}{ Fermeté } \\
\hline & Mois 0 & Mois 3 & Mois 0 & Mois 3 & Mois 0 & Mois 3 & Mois 0 & Mois 3 \\
\hline $\mathrm{HC}$ & $6,0^{a}$ & $5,2^{\mathrm{a}}$ & $4,5^{a b}$ & $2,9^{b}$ & $2,0^{d}$ & $2,6^{d}$ & $2,8^{b}$ & $4,5^{\mathrm{a}}$ \\
\hline $\mathrm{HSHO}$ & $5,9^{a}$ & $5,0^{a}$ & $4,3^{a b}$ & $2,9^{b}$ & $2,5^{\mathrm{cd}}$ & $4,0^{c}$ & $2,9^{b}$ & $4,3^{a}$ \\
\hline CMDG & $3,3^{c}$ & $4,0^{b}$ & $5,0^{\mathrm{a}}$ & $5,0^{a}$ & $3,9^{b}$ & $4,0^{c}$ & $2,3^{c}$ & $2,4^{c}$ \\
\hline MGVL & $4,2^{b}$ & $3,1^{c}$ & $4,0^{b}$ & $2,6^{b c}$ & $3,2^{c}$ & $5,2^{b}$ & $3,6^{a}$ & $3,3^{b}$ \\
\hline SP & $4,0^{b c}$ & $2,7^{c}$ & $3,1^{c}$ & $2,3^{c}$ & $4,8^{a}$ & $6,1^{a}$ & $2,9^{b}$ & $3,1^{b}$ \\
\hline MGVH & $4,4^{b}$ & $3,1^{\mathrm{c}}$ & $3,5^{b c}$ & $2,5^{b c}$ & $2,8^{c}$ & $5,3^{\mathrm{b}}$ & $3,7^{a}$ & $3,7^{\mathrm{ab}}$ \\
\hline
\end{tabular}

${ }^{1}$ Les différentes lettres en exposant indiquent, par colonne, les moyennes significativement différentes $(p=0,05)$

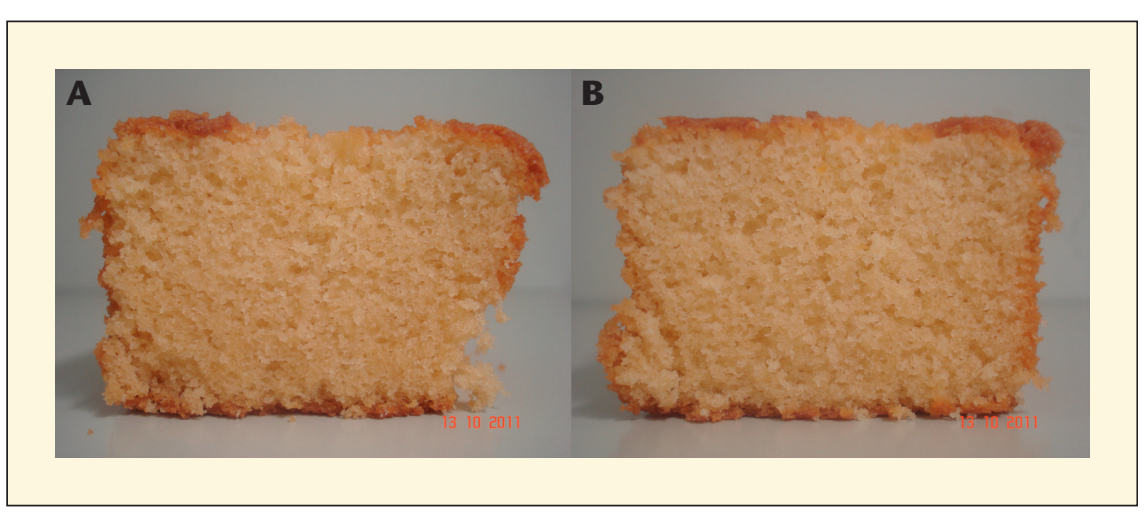

Figure 3. Tranches centrales des cakes obtenus avec le $\mathrm{HSHO}$ réfrigéré à A) $\left.4{ }^{\circ} \mathrm{C}, \mathrm{B}\right)-20^{\circ} \mathrm{C}$.

au cours de la conservation. Ces résultats sont en accord avec Sowmya et al. (2009) et Matsakidou et al. (2010).

L'analyse statistique des résultats obtenus par I'analyse sensorielle descriptive confirme que les 2 séries de fabrication se comportent de façon comparable. Elles ont donc été traitées de façon globale. La couleur et l'aération de la mie ayant été considérées a priori comme des paramètres stables au cours du temps, les résultats des différents points de mesure ont été rassemblés pour ces paramètres. Les profils sensoriels obtenus sur les cakes frais après sélection des paramètres significatifs sont présentés à la figure $2 a$.

L'examen global des profils obtenus permet à nouveau de regrouper les 6 matières grasses en trois groupes : les huiles ( $\mathrm{HC}$ et $\mathrm{HSHO}$ ) d'un côté, les trois matières grasses solides de l'autre, tandis que le CMDG se classe à part. Cette répartition est très claire pour la couleur, I'élasticité, I'humidité et le cassant ; un peu moins pour l'aération, la friabilité et la fermeté.

Pour réaliser les profils sensoriels en fin de durée de vie, seuls les paramètres ayant significativement évolué au cours du temps et montrant une différence significative d'une matière grasse à I'autre sont repris (figure $2 b$ ). À nouveau, les profils obtenus permettent de répartir les 6 matières grasses étudiées dans les 3 groupes présentés ci-dessus.

Le tableau 4 compare les résultats obtenus pour les paramètres significativement influencés par le type de matière grasse en début et en fin de conservation et par la durée de conservation. Le CMDG présente un comportement différent des autres matières grasses : il semble ne pas évoluer en cours de conservation. Les 5 autres matières grasses donnent des évolutions similaires, excepté pour la fermeté, paramètre pour lequel les huiles ( $\mathrm{HC}$ et $\mathrm{HSHO}$ ) se distinguent des 3 matières grasses solides.

L'analyse sensorielle descriptive confirme donc les résultats instrumentaux et permet de classer les 6 matières grasses étudiées dans les 3 groupes décrits plus haut.

Le traitement statistique des résultats de l'analyse hédonique (données non communiquées) sur les cakes frais mon- tre que I'appréciation de la couleur, de la texture et du goût est significativement influencée par le type de matière grasse. En revanche, I'appréciation globale des cakes ne varie pas d'un échantillon à l'autre. Cela a déjà été observé par Dogan et al. (2007).

Ainsi, des différences ont été mises en évidence entre les échantillons (aussi bien selon le type de matière grasse qu'en fonction de la durée de conservation). Celles-ci ont été objectivées par des mesures instrumentales et confirmées par l'analyse sensorielle descriptive. Cependant, ces différences n'impactent pas de façon significative l'appréciation hédonique par le consommateur. Matsakidou et al. (2010) ont déjà mis en évidence des résultats semblables.

\section{Essais complémentaires}

\section{Effet de la température d'incorporation du HSHO}

L'huile de tournesol HSHO mise en œuvre à $12{ }^{\circ} \mathrm{C}$ a donné des résultats très semblables à la référence liquide ( $\mathrm{HC})$. Or cette huile, présentée comme une alternative possible à l'huile de palme, devrait être capable de reproduire le comportement d'une matière grasse solide. De nouveaux essais ont donc été réalisés pour lesquels I'HSHO a été incorporé selon le même protocole mais à 4 et $-20^{\circ} \mathrm{C}$ respectivement plutôt qu'à $12{ }^{\circ} \mathrm{C}$. Visuellement, I'aspect des cakes obtenus avec I'HSHO à 4 ou $-20^{\circ} \mathrm{C}$ se rapproche fortement des cakes obtenus avec les matières grasses solides utilisées dans cette étude (figure 3). Les mesures instrumentales (tableau 5) corroborent ces observations. Le SFC des différentes matières grasses concernées a été estimé à partir des profils de fusion pour les différentes températures de travail 

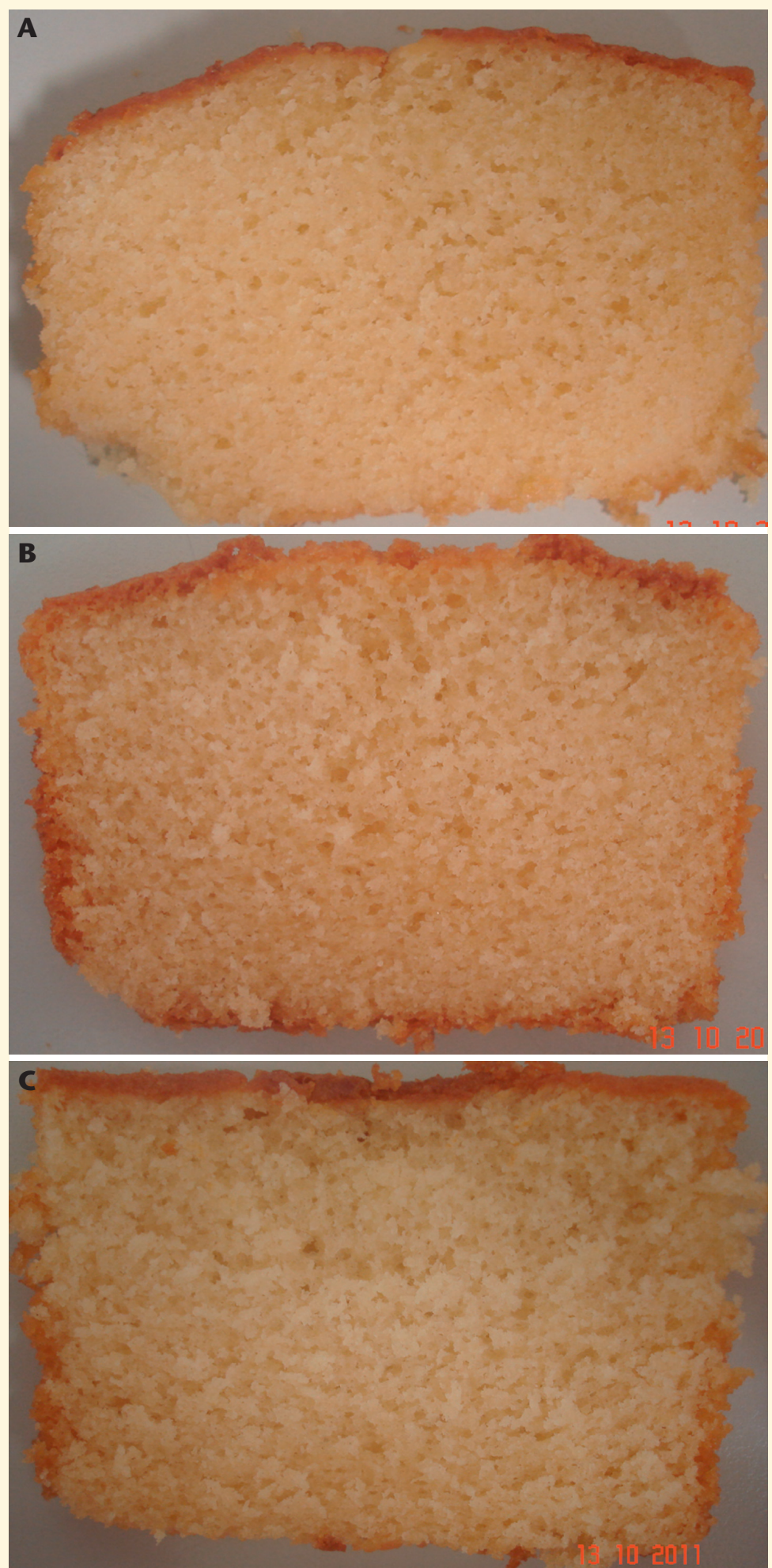

Figure 4. Tranches centrales des cakes obtenus avec différentes proportions des références Colza (HC) et Palme (SP) : A) 75/25 HC/SP, B) 50/50 HC/SP, C) 25/75 HC/SP. (tableau 6). Le SFC résultant du HSHO mis en œuvre à 4 (ou à $-20{ }^{\circ} \mathrm{C}$, bien qu'assez faible, semble suffisant pour influencer la texture et donner un comportement qui se rapproche de celui des matières grasses solides. En revanche, le SFC du HSHO mis en œuvre à $12{ }^{\circ} \mathrm{C}$ devient nul, ce qui explique pourquoi il se comporte alors comme la référence liquide.

\section{Effet du SFC}

L'huile de colza et le shortening à base d'huile de palme ont ensuite été combinés dans des proportions variables. Les deux matières grasses sont incorporées séparément (pas de pré-mélange). Le SFC des différentes combinaisons a été calculé pour les différentes températures de travail à partir des profils de fusion des matières grasses pures. Une première observation des cakes obtenus (figure 4) permet de constater que la texture de la mie et le profil des tranches s'éloignent progressivement des caractéristiques obtenues avec I'huile de colza pour se rapprocher progressivement de celles obtenues avec le shortening pur lorsque la proportion de shortening augmente. L'analyse instrumentale de la texture confirme ces observations (tableau 7). Ces tendances confirment celles observées par Sowmya et al. (2009) et Matsakidou et al. (2010). On peut considérer que l'on retrouve les caractéristiques obtenues avec le shortening pur dès la proportion 50/50 Colza/ Shortening, ce qui correspond à un SFC mis en œuvre de $5 \%$ et un SFC résultant dans la pâte estimé à $12 \%$ (tableau 8). Ces résultats tendent donc à montrer qu'il est effectivement indispensable de conserver une fraction solide pour retrouver les paramètres de texture obtenus avec une matière grasse solide, mais qu'il est sans doute possible de réduire ce $S F C$ à un minimum nécessaire (situé autour de $5 \%$ à $20-25^{\circ} \mathrm{C}$ par Bennion et Bamford (1997)). Ceci permettrait en effet de réduire la proportion de matières grasses saturées (palme notamment) dans les shortenings, ce qui contribuerait à I'amélioration de leurs profils nutritionnels. Ces résultats expliquent aussi les observations réalisées pour le HSHO : malgré le SFC faible de cette matière grasse, le seuil minimal requis pour obtenir une texture du type de celle 
Tableau 5. Caractérisation des cakes obtenus avec le HSHO réfrigéré et comparaison avec les références Colza et Palme

\begin{tabular}{|llllll|}
\hline & Dureté & Élasticité & Gumminess (N) & Cohésion & Hauteur (cm) \\
\hline $\mathrm{HC} 12{ }^{\circ} \mathrm{C}(\mathrm{n}=3)$ & $5,7 \pm 0,7$ & $0,87 \pm 0,02$ & $3,1 \pm 0,3$ & $0,54 \pm 0,01$ & $6,3 \pm 0,4$ \\
\hline $\mathrm{HSHO} 12{ }^{\circ} \mathrm{C}(\mathrm{n}=3)$ & $5,6 \pm 0,7$ & $0,83 \pm 0,04$ & $3,0 \pm 0,4$ & $0,55 \pm 0,01$ & $5,9 \pm 0,6$ \\
\hline $\mathrm{HSHO} 4{ }^{\circ} \mathrm{C}(\mathrm{n}=2)$ & $4,4 \pm 0,2$ & $0,79 \pm 0,02$ & $2,4 \pm 0,1$ & $0,54 \pm 0,01$ & $5,8 \pm 0,3$ \\
\hline $\mathrm{HSHO}-20^{\circ} \mathrm{C}(\mathrm{n}=2)$ & $4,1 \pm 0,1$ & $0,76 \pm 0,03$ & $2,1 \pm 0,0$ & $0,54 \pm 0,01$ & $5,6 \pm 0,3$ \\
\hline $\mathrm{SP} 37^{\circ} \mathrm{C}(\mathrm{n}=3)$ & $5,1 \pm 1,0$ & $0,76 \pm 0,05$ & $2,6 \pm 0,5$ & $0,51 \pm 0,02$ & $5,3 \pm 0,3$ \\
\hline
\end{tabular}

Tableau 6. Teneurs en fraction solide de HC, HSHO et SP aux différentes températures de travail

\begin{tabular}{|c|c|c|c|c|c|}
\hline & HC & $\begin{array}{l}\text { HSHO } \\
12{ }^{\circ} \mathrm{C}\end{array}$ & $\begin{array}{l}\text { HSHO } \\
4{ }^{\circ} \mathrm{C}\end{array}$ & $\begin{array}{l}\text { HSHO } \\
-20{ }^{\circ} \mathrm{C}\end{array}$ & SP \\
\hline Température de la matière grasse avant incorporation & $12^{\circ} \mathrm{C}$ & $12^{\circ} \mathrm{C}$ & $4{ }^{\circ} \mathrm{C}$ & $-20^{\circ} \mathrm{C}$ & $37^{\circ} \mathrm{C}$ \\
\hline SFC correspondant & $0,0 \%$ & $14,0 \%$ & $23,0 \%$ & / & $10,0 \%$ \\
\hline Température de la pâte avant incorporation & $24{ }^{\circ} \mathrm{C}$ & & & & \\
\hline SFC correspondant & $0,0 \%$ & $0,0 \%$ & $0,0 \%$ & $0,0 \%$ & $24,0 \%$ \\
\hline Température de la pâte après incorporation & $22{ }^{\circ} \mathrm{C}$ & $22^{\circ} \mathrm{C}$ & $18^{\circ} \mathrm{C}$ & $15^{\circ} \mathrm{C}$ & $25^{\circ} \mathrm{C}$ \\
\hline SFC correspondant & $0,0 \%$ & $0,0 \%$ & $3,5 \%$ & $6,0 \%$ & $22,0 \%$ \\
\hline
\end{tabular}

Tableau 7. Caractérisation des cakes obtenus avec différentes proportions des références Colza (HC) et Palme (SP)

\begin{tabular}{|llllll|}
\hline $\mathbf{n = 3}$ & Dureté $(\mathbf{N})$ & Élasticité & Gumminess $(\mathbf{N})$ & Cohésion & Hauteur $(\mathbf{c m})$ \\
\hline HC $100 \%$ & $5,7 \pm 0,7$ & $0,87 \pm 0,02$ & $3,1 \pm 0,3$ & $0,54 \pm 0,01$ & $6,3 \pm 0,4$ \\
\hline HC/SP 75/25 & $5,9 \pm 0,2$ & $0,81 \pm 0,01$ & $3,3 \pm 0,1$ & $0,55 \pm 0,01$ & $5,5 \pm 0,1$ \\
\hline HC/SP 50/50 & $5,3 \pm 0,4$ & $0,77 \pm 0,03$ & $2,8 \pm 0,1$ & $0,54 \pm 0,01$ & $5,3 \pm 0,2$ \\
\hline HC/SP 25/75 & $4,1 \pm 0,3$ & $0,75 \pm 0.03$ & $2,0 \pm 0,2$ & $0,52 \pm 0,01$ & $5,1 \pm 0,3$ \\
\hline SP 100\% & $5,1 \pm 1,0$ & $0,76 \pm 0,05$ & $2,6 \pm 0,5$ & $0,51 \pm 0,02$ & $5,3 \pm 0,3$ \\
\hline
\end{tabular}

Tableau 8. Teneurs en fraction solide des différents mélanges des références Colza $(H C)$ et Palme (SP) aux températures de travail

\begin{tabular}{|c|c|c|c|c|c|}
\hline & HC $100 \%$ & $\mathrm{HC} / \mathrm{SP} 75 / 25$ & HC/SP 50/50 & HC/SP 25/75 & SP $100 \%$ \\
\hline Température de la matière grasse avant incorporation & $12^{\circ} \mathrm{C}$ & $12^{\circ} \mathrm{C} / 37^{\circ} \mathrm{C}$ & $12^{\circ} \mathrm{C} / 37^{\circ} \mathrm{C}$ & $12^{\circ} \mathrm{C} / 37^{\circ} \mathrm{C}$ & $37^{\circ} \mathrm{C}$ \\
\hline SFC correspondant & $0,0 \%$ & $2,5 \%$ & $5,0 \%$ & $7,5 \%$ & $10,0 \%$ \\
\hline Température de la pâte avant incorporation & $24^{\circ} \mathrm{C}$ & & & & \\
\hline SFC correspondant & $0,0 \%$ & $6,0 \%$ & $12,0 \%$ & $18,0 \%$ & $24,0 \%$ \\
\hline Température de la pâte après incorporation & $22^{\circ} \mathrm{C}$ & $24^{\circ} \mathrm{C}$ & $24^{\circ} \mathrm{C}$ & $25^{\circ} \mathrm{C}$ & $25^{\circ} \mathrm{C}$ \\
\hline SFC correspondant & $0,0 \%$ & $6,0 \%$ & $12,0 \%$ & $16,5 \%$ & $22,0 \%$ \\
\hline
\end{tabular}

obtenue avec une matière grasse solide est atteint lorsqu'on le réfrigère.

\section{Conclusion}

Six matières grasses ont été étudiées : une référence liquide (colza - $\mathrm{HC}$ ), une référence palme (shortening - SP), deux margarines (I'une à base de matière grasse laitière - MGVL - et l'autre à base de matières grasses végétales hydrogénées - MGVH), un système à base d'huile de colza épaissie avec des émulsifiants (CMDG), et une huile de tournesol HSHO. Sans surprise, de fortes différences ont été mises en évidences. Les résultats instrumentaux et I'analyse sensorielle descriptive ont permis de classer les matières grasses étudiées en 3 " familles ": les matières grasses liquides (colza et tournesol HSHO) donnent des résultats similaires, les trois matières grasses solides (shortening et margarines) 
montrent les mêmes comportements, et enfin, le système émulsifié se classe à part.

Le tournesol HSHO (Nutrisun) est présenté par ses fabricants comme une nouvelle solution pouvant se substituer à I'huile de palme. Incorporée à température ambiante ou à $12^{\circ} \mathrm{C}$, il $s^{\prime}$ avère que cette huile se comporte en réalité comme l'huile de colza, donc comme une matière grasse liquide. En revanche, incorporé à plus basse température $\left(4{ }^{\circ} \mathrm{C}\right.$ ou $\left.-20^{\circ} \mathrm{C}\right)$, le comportement du Nutrisun tend à se rapprocher de celui des matières grasses solides.

Le système émulsifié (CMDG) donne une texture qui semble peu adéquate. En revanche, les cakes produits avec cette matière grasse sont très stables dans le temps. Cela met en évidence le rôle des émulsifiants dans l'obtention et la conservation d'une texture moelleuse et la place qu'ils pourraient occuper dans l'optimisation des formulations (Delacharlerie et al., 2004 ; Cauvain et Young, 2006).

Des différences ont donc été observées entre les 6 matières grasses étudiées aussi bien par voie instrumentale que par établissement du profil sensoriel. Cependant, I'analyse hédonique n'a pas permis de montrer une différence dans I'appréciation globale des différentes recettes. Cela peut signifier (à confirmer avec un jury plus large) que le consommateur n'est pas forcément attaché à la texture obtenue avec I'huile de palme (ou autre matière grasse solide), et qu'il est donc possible de lui proposer autre chose, avec une texture différente, qui pourrait avoir le même succès.

Cette étude montre donc que l'huile de palme donne effectivement des propriétés sensorielles particulières, mais n'est pas indispensable. Elle peut, sans impact sensoriel majeur, être remplacée par d'autres matières grasses solides. Elle peut aussi, sans forcément perturber le consommateur, être remplacée par une huile liquide.

Les résultats obtenus confirment également que la texture n'est pas conservée si la fraction solide est supprimée. Cependant, il a été montré qu'une texture acceptable pouvait être obtenue avec un SFC assez faible (aux alentours de $10 \%$ - voire $5 \%$ - à la température de la pâte). Cela signifie que le SFC des shortenings actuels (20-25\%) pourrait franchement être diminué. Il y a donc une réelle opportunité à étudier l'optimisation des mélanges de corps gras de façon à améliorer leurs profils nutritionnels (diminution du taux d'acides gras saturés). Dans cette optique, le fractionnement, l'inter-estérification et le mélange des corps gras sont des voies à exploiter (Dogan et al., 2007 ; Matsakidou et al., 2010; Morin et Goullieux, 2011), de même que la technologie des émulsifiants et I'utilisation opportune de certains hydrocolloïdes (Ghotra et al., 2002 ; Sowmya et al., 2009)

Il faut toutefois garder à l'esprit que les résultats présentés ici sont valables pour une famille de produits proches de celui étudié, mais ne peuvent pas être transposés tels quels à d'autres applications : cela devrait faire l'objet d'études séparées. Le travail d'optimisation des shortenings en vue de la diminution de la teneur en acides gras saturés doit être réalisé application par application.

Remerciements. Cet article a été rédigé dans le cadre du projet Nutrisens émargeant au programme Interreg IV France-Wallonie et cofinancé par le Fonds Européen de Développement Régional (FEDER), le Service Public Wallonie, la Communauté Urbaine d'Arras et la Région Nord - Pas de Calais Les auteurs tiennent également à remercier Mesdemoiselles Marjorie Servais et Farah Darbouche pour leur appui technique.

\section{RÉFÉRENCES}

Bennion EB, Bamford GST. The technology of cake making $6^{\text {th }}$ ed. Londres : Blackie Academic \& Professional, 1997.

Berger KG, Idris NA. Formulation of zerotrans acid shortenings and margarines and other food fats with products of the oil palm. JAOCS $2005 ; 82$ : 775-81.

Bourne MC. Food texture and viscosity: concept and measurement. New York : Academic press, 2002.

Cauvain SP, Young LS. Baked Product: Science, Technology and Practice. USA : Wiley \& Sons, 2006.

Chueamchaitrakun P, Compreeda P, Haruthaithanasan V, Suwonsichon T, Kasemsamran S, Prinyawiwatkul W. Sensory descriptive and texture profile analyses of butter cakes made from composite rice flours. Int J Food Sci Tech $2011 ; 46$ : 2358-65.

Corley RHV. How much palm oil do we need? Environ Sci Policy 2009 ; 12 : 134-9.

Conseil Supérieur de la Santé. Recommandations nutritionnelles pour la Belgique. Bruxelles : SPF Santé Publique, Sécurité de la Chaîne alimentaire et Environnement, 2009.

Delacharlerie S, Blecker C, Kegelaers Y, Deroanne C. Les émulsifiants dans les produits de boulangerie industrielle : modes d'action, rôles et utilisations. Industries Alimentaires et Agricoles 2004, Mai : 8-14.

de Moraes Mizurini D, da Costa Maia I, de Carvalho Sardinha FL, Monteiro RQ, OrtizCosta S, do Carmo MG. Venous thrombosis risk: effects of palm oil and hydrogenated fat diet in rats. Nutrition $2011 ; 27$ : 233-8.

Dogan IS, Javidipour I, Akan T. Effects of interesterified palm and cottonseed oil blends on cake quality. Int / Food Sci Tech 2007 ; 42 : 157-64.

Fernandez-Moya V, Martinez-Force E, Garcès R. Oils from improved high stearic acid sunflower seeds. Journal of Agriculture and Food Chemistry 2005 ; 53 : 5326-30.

Fitzherbert EB, Struebig MJ, Morel A, et al. How will oil palm expansion affect biodiversity? Trends Ecol Evol 2008 ; 23 : 538-45.

Ghotra BS, Dyal SD, Narine SS. Lipid shortenings : a review. Food Res Int 2002 ; 35 : 1015-48.

Graille J, Pina M. L'huile de palme : sa place dans I'alimentation humaine. Plantations, recherche, développement 1999; (MarsAvril) : 85-90.

Greenpeace. How the palm oil industry is cooking the climate. Greenpeace International, 2007.

Kazier $\mathrm{H}$. Palm oil versatile ingredient solution to challenge bakery applications. $5^{\text {th }} \mathrm{global}$ oils and fats business forum. Las Vegas, 2007.

Koh LP, Wilcove DS. Is oil palm agriculture really destroying tropical biodiversity? Conservation Letters $2008 ; 1: 60-64$.

Kummerow FA. The negative effects of hydrogenated trans fats and what to do about them. Atherosclerosis $2009 ; 205$ : 458-65.

Legrand P. Intérêt nutritionnel des acides gras saturés. Sci Aliment $2010 ; 29$ : 115-22.

Martin A. Apports nutritionnels conseillés pour la population française, $3^{\mathrm{e}}$ édition. Paris : Tec \& Doc, Lavoisier, 2001.

Matsakidou A, Blekas G, Paraskevopoulou A. Aroma and physical characteristics of cakes prepared by replacing margarine with extra olive oil. Food Sci Technol 2010; 43 : 949-57. 
Morin O, Goullieux I. Formulations de matières grasses pour biscuiterie et feuilletages industriels : intégrer impératifs technologiques, qualités organoleptique et nutritionnelle. Industries Alimentaires et Agricoles 2011 ; 05/06 : 34-5.

Pernetti M, van Malssen K, Kalnin D, Floter E. Structuring edible oil with lecithine and sorbitan tri-stearate. Food Hydrocolloid 2007 ; 21 : 855-61.

Rogers MA. Novel structuring strategies for unsaturated fats - Meeting the zero-trans, zero-saturated fat challenge: a review. Food Res Int $2009 ; 42$ : 747-53.

Sowmya M, Jeyarani T, Jyotsna R, Indrani D. Effect of replacement of fat with sesame oil and additives on rheological, microstructural, quality characteristics and fatty acid profile of cakes. Food Hydrocolloïd $2009 ; 23$ : 1827-36.

Walrand S, Fisch F, Bourre JM. Tous les acides gras saturés ont-ils le même effet métabolique? Nutr clin Metab 2010 ; 24 : 63-75. 